Volume 8, No.5, September - October 2019

International Journal of Advanced Trends in Computer Science and Engineering

Available Online at http://www.warse.org/IJATCSE/static/pdf/file/ijatcse18852019.pdf

https://doi.org/10.30534/ijatcse/2019/18852019

\title{
Dual Parametric Stabilization of Interference and Throughput in Wireless Sensor Network- Optical Communication
}

\author{
Akshatha Hari Bhat ${ }^{1}$, Dr. H V Balachandra Achar Author ${ }^{2}$ \\ ${ }^{1}$ Vidyalankar Institute of Technology, Mumbai \\ ${ }^{1}$ Research Scholar, Shri Madhwa Vadiraja Institute of Technology and Management, Bantakal, Udupi \\ akshathahbhat@gmail.com \\ ${ }^{2}$ Shri Madhwa Vadiraja Institute of Technology and Management, Bantakal, Udupi, hvbachar@gmail.com
}

\begin{abstract}
Self-adapting wireless communication in data exchange has gained a lot of interest and importance in the recent past. For monitoring and controlling of vital physical parameters Wireless Sensor Network (WSN) has evolved. The property of WSN to link to itself is very useful and this acts as an advantage under critical access zones. With the significance of using interface in a wireless mode and easy deployment, WSNs are now used in various real-time monitoring applications. However, these networks are bounded with the need of accuracy and transmission throughput to perform efficiently in real-time applications. Wherein the new hybrid techniques such as integrated optical communication offer high communication spectrum, still the accuracy and throughput are always the dual problems. In this paper, we present an optimal communication approach in WSN interface to Optical Communication based on adaptive light modulation for minimal interference and maximum throughput. The proposed solution is focused to develop a spectrum allocation in WSN Optical Communication model with respect to offered service rate and the interference margin. The dynamic behavior of modulation control, leads to a higher resource utilization, lower computation overhead, higher accuracy and higher offered throughput.
\end{abstract}

Key words: Dual problem, Dynamic Modulation high throughput, Optical Communication (OC), Wireless Sensor Network (WSN)

\section{INTRODUCTION}

The revolutionary evolution in the area of wireless communication has developed many new communication approaches for data exchange which leads to high resource utilization and offers greater system efficiency. Systems such as hybrid network, heterogeneous network, cognitive network, Ad-hoc network, WSN are a few of these advanced networks. Each of these networks has given a boost to resource utilization, throughput, data accuracy, service compatibility, etc. Among these approaches, WSNs are now been developed for various critical monitoring applications, such as the power plants, medical data monitoring, distributed industries, military applications, corporate applications etc. WSNs represent a new mode of communication in wireless technology with significant interest in remote and industrial applications. WSNs offer exclusive benefits and compatibility in case of low-power and low-cost rapid positioning applications that do not need human supervision. Some of these applications include disaster recovery, military surveillance, health administration, environmental \& habitat monitoring, target-tracking, etc. Besides these advantages, to utilize a WSN efficiently, there is a need of an efficient routing protocol, which plays an important role in the data transfer from sensors. The sensors in WSN also have limited resources like limited bandwidth, limited power and inconstant network topology with data redundancy. In WSN, every node has multiple paths to exchange information with another node and also acts as a router to send information from one node to another. The nodes route through various nodes, increasing the throughput of the entire network by sending multiple information at a time through multiple paths. Multipath routing strategy can prolong the life span of network by balancing the energy consumption of each node by shunting messages through multipath. Multiple routing strategies further improve the stability and reliability of the network. Therefore, it can provide much better service for network applications. In addition to the advantages of multipath routing, the issue of congestion is predominant, resulting in the bottle neck problem which leads to the decrement in throughput of the system. The advantage of wireless deployment and dynamic routing/linking gets disrupted due to dynamic trafficking and hence leads to decrease in system throughput. For the enhancement of resource optimization and throughput, optical networks are integrated to offer higher optical spectrum.

In the approach of exchanging data in optical domain for sensor network, Zuhurul et al [1] presented an approach to real-time data exchange. The recent development in the field of micro grid system is applied with sensor nodes and integrated with optical communication for data exchange among different nodes and such application is presented using different modes of communication, such as ZigBee, Wi-Fi, Wi-Max and cellular network. Alwis et al [2] presented the mode of communication in sensor network which is termed as optical fiber sensor (OFS). This approach is observed to be an emerging area of communication where long range communication is possible with the usage of optical signal 
with sensor nodes interfaced. This network has a large capacity of data transferring hence very suitable in next generation communication. Not much of developments are observed and hence, the prospect of developments exists in multiple domains. Villatoron \& Zubia [3] proposed a technique where different types of sensor devices and communication structures are emphasized for future generation OFS communication. A new format of OFS using photonic crystal fiber sensors (PCF) is outlined by Xenakis et al [4]. The integration of PCF with sensor nodes could be an optimal solution for demanding raise of data exchange for future generation sensor node optical communication. It also requires optimization of various parameters of communication such as power, topology and interface. Liao et al [5] presented a simulated annealing (SA) approach, which optimizes the power consumption based on the sensor node deployment and its energy model. For energy conservation various approaches were proposed in past. The cluster based scheme is developed to realign the network distribution for power saving which is presented by Sun et al [6]. Harshitha Chaurasiya \& Dr. Shivnath Ghosh also provided brief study on cluster based routing protocol [7]. The clustering approach rearranges the node distribution, to achieve the power conservation. As the power is heavily drained under high traffic condition, to control the power conservation under high interference cross layer protocol is defined by Naderi et al [8] which performs monitoring of data exchange among the nodes to control power dissipation using high traffic condition. Simpson et al [9] presented an approach of underwater optical communication using a low cost test platform. The system reliability is enhanced with the usage of a digital signal processing with error correction approaches. The communication is defined by the interface of an optical sensor with a sensor node placed under water. Zahir Ahmad \& Roger Green [10] outlined the method of optical communication using green/blue light emitting diode (LED). The approach defined the significance of long range communication using Multi-hop communication in comparison to a single hop communication. The approach is made compatible for a bandwidth of $100 \mathrm{KHz}$ to a range of one meter in control of accuracy and signal to noise ratio. Saeed et al [11] presented energy conservation using harvesting approach. Energy is observed to be a constraint in optical communication as the installations of these communication units are located at remote locations or underwater. Batteries are the only and limited source of energy. Energy harvesting based on the signal strength of received signal is proposed. An analysis of energy coding using Cramer Rao Lower Bound (CRLB) on a benchmark is evaluated for the localization performance. A similar approach of energy conservation was made by $\mathrm{Na}$ Zhang [12]. This approach defines an optical efficient energy multi path routing protocol for free space optical communication. The approach conserves the energy per node to improve the life time of the network. This approach improves the energy conservation over large network topology. Gomes et al [13] proposed radio-optical fiber communication. The proposed approach is validated for different wireless personal area network such as the ZigBee and ultra-wideband network. This proposed approach is validated over standard communication protocol of radio-fiber communication. Afzal et al [14] presented a line of sight and Quasi diffuse communication. The paper defines an approach of multi spot quasi diffuse beam communication for optical wireless communication. A modified Corner Cube Reflector (CCR) and thin film CCR is suggested for data exchange and sensor node charging operation. The approach illustrated a higher communication efficiency and longer lifetime of the network. The deployment of optical wireless communication at indoor location is outlined by Elgala et al [15]. The main focus is on the issue of deployment under duplex mode of communication. A MAC protocol for link capacity improvement is presented. The indoor deployment using LED based optical wireless communication is presented by Lee et al [16]. Here, each sensor node is interlinked to a server through power line communication and Ethernet linking. This approach defines a full duplex mode of communication and optimizes the inter-symbol interference during the data exchange. In real-time application to optical communication in wireless mode, Chinrungrueng et al [17] presented traffic monitoring. The integrated model of wireless sensor network and optical network efficiently monitors the traffic condition and alerts the commuter the congestion condition. Shota Teramoto \& Tomoaki Ohtsuki [18] presented CCR based approach using an ON-OFF keying (OOK). Tow decision approach for signal detection using collective and majority decision is presented. In collective decision approach, the buffered signals are hard threshold for a decision. Whereas in majority decision results for photo detector depends on the sensors hard detection and the maximum potential defines the decision value. The BER performance in this case is comparatively improved for large number of sensor nodes. An integrated design of optical and wireless communication is presented by Yang et al [19]. Here, the technique develops a fiber Bragg grating approach using temperature sensors with minimal power utilization. The integration approach illustrated, gave an optimal enhancement in communication performance in data exchange compared to optical or WSN communication alone. Li et al [20] presented a free-space optical communication to cover the backhaul in cellular network. A linear integer programming model is proposed in FSO design. A mirror component is used in the linear program to detect k-disjoint paths in the network, which results in more scalability in the network. Among the communication approach, the light modulation has a limitation of interference due to refraction and reflection of optical beam over the fiber units. In traffic flow model, buffer management is applied. Rao et al [21] defined few node placement problems and suggested feasible path for communication. The cross layer optimization of service stream, data traffic at router level was proposed by Taruna et al [22]. The approach of coding was introduced at network abstraction layer (NAL), where the buffer based congestion control following buffer Management and relative Quality of Service (QoS) was mapped to schedule the rate of data traffic flow. Among the communication approach, the light modulation has limitation of interference due to refraction and reflection of optical beam over the fiber units. It 
is hence required to develop an adaptive modulation for spectrum allocation in optical means for interference controlling and throughput enhancement.

To present the proposed approach, the rest of the paper is outlined in 7 sections. The approach of wireless sensor network communication is outlined in 2 . Section 3 presents the conventional optical modulation scheme for WSN-optical communication. Section 4 presents the proposed adaptive interference mitigation in WSN-OC architecture. Simulation result for the developed approach is outlined in section 5 . Section 6 outlines the conclusion for the developed approach.

\section{WSN COMMUNICATION}

WSNs are deployed extensively in physical monitoring and have spread through wide area network for observing the necessary parameters. They are deployed in remote location to collect data and transmit over a long range to the monitoring point. WSNs are used in many applications for power conservation and rapid deployment in the field of monitoring application. WSN has a significant usage in various real time applications. However, due to the increase in the requirement of WSNs, for various new applications, the demands of new routing protocol, security measure, power conservation are also increasing. Many of the applications demand a high rate transmission with optimal resource utilization. An existing communication model of wireless sensor network is illustrated in figure 1.

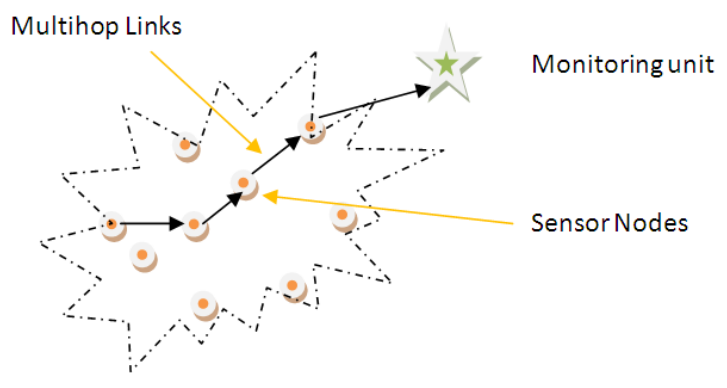

Figure 1: Network deployment in wireless sensor network

Sensors are deployed at remote monitoring points which are interfaced with transceivers. Each of the nodes is designed to read its side neighbors, discover routes for data exchange and conserve energy for longer life time. In a long range communication, the nodes are interlinked and form a Multi-hop environment to exchange data over a long range communication. A source discovers all possible routes and selects an optimal and shortest path offering highest bandwidth. This makes the wireless sensor interface more efficient for industrial and field applications. The sensor node communications are used as optimal routing nodes to exchange data over a wide area. Accuracy and offered throughputs are the primary needs in this network. To achieve a higher throughput, WSNs are integrated with optical communication because it offers high bandwidth.

\section{WSN OPTICAL MODULATION INTERFACE}

The higher bandwidth and dedicated medium of data exchange have made optical communication as one of the optimal modes of data exchange for upcoming communication demands. The dedicated medium offers a secure path of data exchange with light as a source of communication. In the recent past, optical communications are explored as a possible mode of communication in a hybrid model where, two or more independent networks offering resource potential are interlinked for data exchange. This hybrid network can be used in the situation that demands higher throughput as it offers higher data rate and security. In different network communication, WSN has a critical demand of higher throughput due to its application of highly sensitive data monitoring. Here, the sensed signals are needed to be transmitted to the monitoring center at highest rate. Each of the sensed signals is to be refreshed at a very faster rate for monitoring. Optical Communication has high bandwidth and hence is suitable in this scenario. To exchange sensor data over a WSN with optical fiber interconnects, a hybrid mode of data exchange is required. A general interface approach is illustrated in figure 2 .

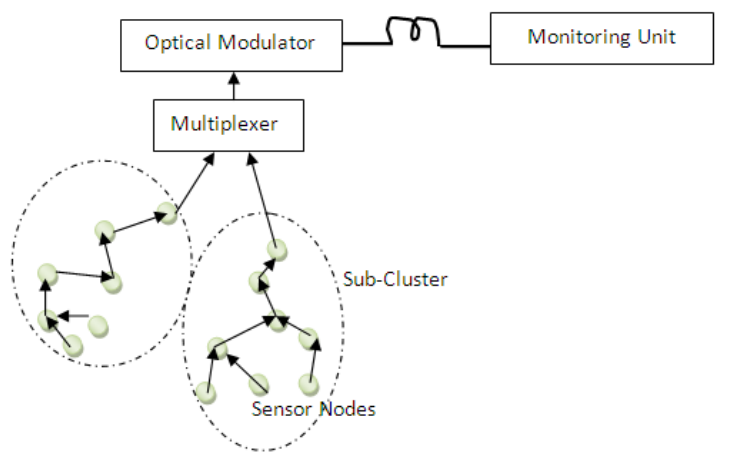

Figure 2: Interfacing of WSN-Optical Fiber Communication

Here the sensor network nodes used for sensing data are interfaced using sensor devices, encoded and transmitted over a wireless network. The wireless communication is made for a sub cluster zone with nodes placed at closer proximity. Each of the clusters is linked to a centralized node, wherein the signals are transmitted using optical fiber for long range communication. Each of the nodes in WSN is interfaced in sub cluster forming multiple sub-clusters, where each sub cluster is interlinked to an optical link to transport to long range monitoring unit. This mode of exchange results in faster data transmission, and hence improves the rate of data updation at monitoring terminal. The exchange of multiple data simultaneously improves the system throughput. However, the transmitting signal observes a significant interference in the signal. In the optical mode communication, the estimation operation of the signal received is performed based on the thresholding of the magnitude value. The decisions are erroneous under dynamic interference model. Hence, in this 
paper an optimal estimation based on adaptive threshold margin is proposed.

\section{INTERFERENCE MITIGATION APPROACH}

There is a large self-created interference observed at the source side. These noises are to be eliminated as it could result into multiplicative noise during communication in light propagation. To minimize the noise effect, a new threshold logic based interference minimization technique has been developed. This approach minimizes the noise level based on the derived noise margin developed from the discrete input value. In the signal propagation when noise is added it illustrates a random pattern. To detect the available signal and the noise level, the signal is sampled in a discrete manner and the average energy variation is considered. The average energy margin is considered as a noise margin and the signal potential below the threshold level is considered as a detected signal else suppressed as noise.

In the modeled communication approach of integrated WSN and optical fiber, each of the sensed data is modulated using optical signal with light intensity controlled by the modulating wavelength. In the communication process, at the source of generation and at the refraction of the bends, the optical signals are scattered or electrical noise at the source encoding gets added which is observed as noise, which affects the signal estimation at the receiver. In the process of signal reception, a photo detector is used to measure the signal to noise strength $(\mathrm{S} / \mathrm{N})$ for detection of potential value of ' 1 ' or ' 0 ' at the receiver. The existing detectors set a limit to the measured potential and a logical ' 1 ' or ' 0 ' is observed as a result of the thresholding of the limited value. The signal to noise ratio is defined as,

$$
\frac{s}{N}=\frac{\text { Detected aignal power by photodetector }}{\text { Noise power of Photodetector }+ \text { Noise amplifier }}
$$

The noise margin is considered to be constant in existing approach and a matched filter is used for the thresholding of the received potential. However, due to variant sources and multiple bends in the fiber there is considerable deviation in the signal strength. A matched filter approach in this case increases the estimated performance for variable magnitude of the received signal. Here the photo detector at receiver end converts the photon-to-electron and a thermal noise is observed at the amplifier unit. For a higher estimation performance the signal-to-noise ratio is to be high which demands for a high quantum efficiency with lower amplifying noise. The observed energy is the difference of the two energy states of higher and lower levels as $\mathrm{E}_{2}, \mathrm{E}_{1}$ respectively. The energy difference is defined as;

$$
E=E_{2}-E_{1}=h v
$$

Here, ${ }^{\prime \prime} h^{x}$ is the plank constant and ${ }^{n} v^{\prime}$ is light velocity.
Here a dynamic threshold is developed which is adjusted adaptively using the measured potential of current and past observation.

In the detection process, each of the received signals is discretized using sampling factor $(\mathrm{S})$. Each of the samples is then taken as threshold. An aggregated threshold value for a bit period is counted and a decision of ' 1 ' or ' 0 ' is taken. The description of the decision logic is shown in figure 3 .

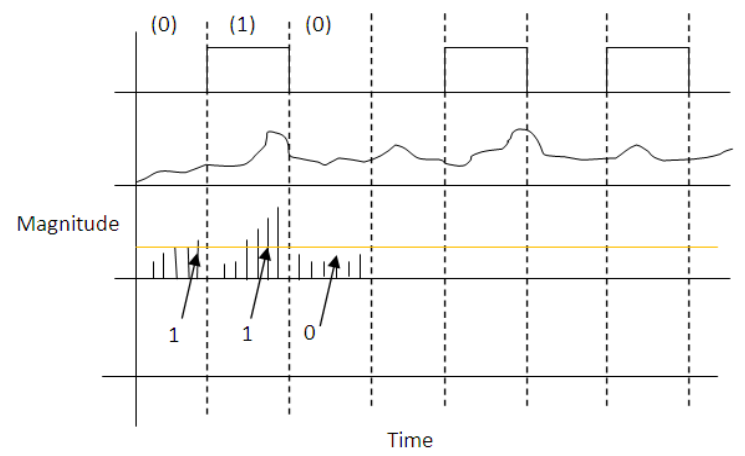

Figure 3: Existing thresholding approach

Wherein the conventional approach has a fixed threshold, 1 single coefficient crossing the threshold is read as 1 else 0 . The dynamic variation of the noise level gives a wrong decision of ' 1 ' or ' 0 ' logic. To improve the estimation performance, a dynamic threshold approach is developed. This approach observes a decision value for a monitoring period say, ' $t$ '. The majority count value of potential high or low is then computed. The majority count value is set as the current threshold value. On reception of next processing block, the signals are processed to sample the signal and measure the majority. If the current majority count is higher than the past value, the current majority value is considered as a new threshold else the previous value remains intact. This process results in a dynamic setting of threshold margin based on the current limiting and past recorded value. Here, for a linear variation with constant noise effect the threshold set is continued. Whereas for a random variation, each time the threshold is computed and compared for updating. The thresholding and decision process is illustrated in figure 4 .

Figure 4: Proposed approach of decision logic

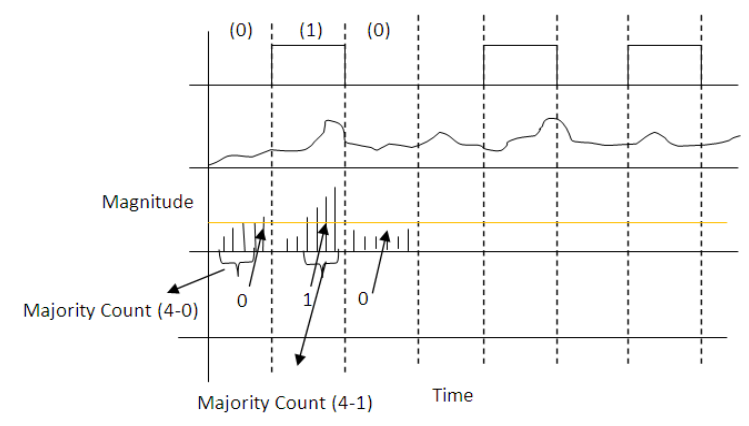


Here, for a transmitted bit pattern in ' 1 ' and ' 0 ' shown in figure 4(a), the transmitted signal is distorted in magnitude as illustrated in figure 4(b). The threshold approach individually separates and distinct the signal and count the majority value for a decision. In the $2^{\text {nd }}$ recursion the threshold is set based on the previous threshold and new count value. The estimated signal is observed at figure 4(d). This approach decreases the misclassification probability under dynamic variation and hence results in more accurate estimation. The estimation performance is measured and the simulation model is presented in the next section.

\section{SIMULATION RESULT}

Simulation model of the proposed approach is developed as an integrated unit of distributed sensor nodes and link nodes with optical fiber links for data exchange. For the simulation of the proposed approach following network parameters are considered.

Table 1: Network Parameter for Simulation

\begin{tabular}{|c|c|}
\hline Network parameter & Characteristic \\
\hline Network Layout & Random \\
\hline No. of Nodes & 30 \\
\hline Network area & $120 \times 120 \mathrm{~m}^{2}$ \\
\hline Link node range & $25 \mathrm{~m}$ \\
\hline
\end{tabular}

The simulated network layout is shown in figure 5. Each of the sensor nodes in the network is deployed with a sensor and a transponder unit to sense the physical parameter and is encoded for transmission.

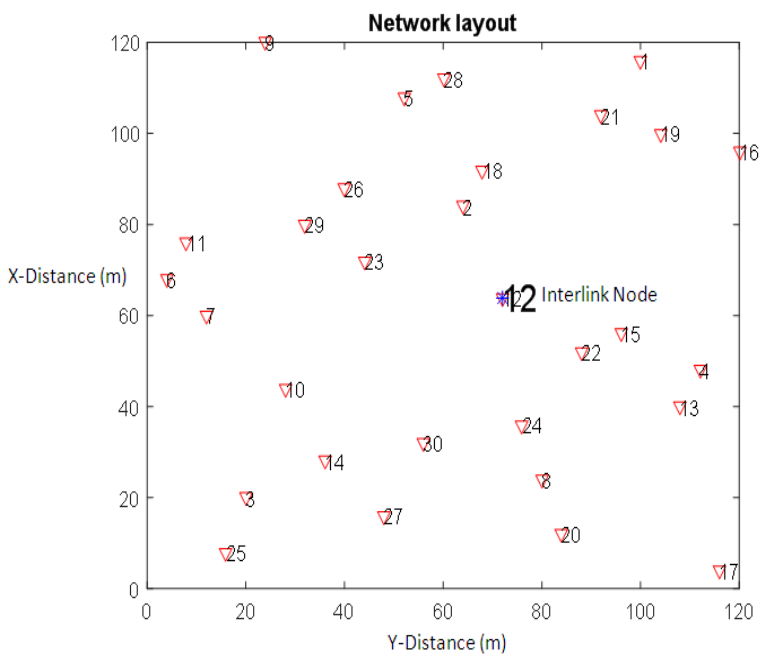

Figure 5: Simulation Network Layout

In communication process, each of the nodes is processed for its link establishment, where each link discovers its neighbor by handshaking a broadcast packet within a communication range. Each of the nodes is linked with a side node and formulates sub clusters for communication. While exchanging the data packet from source to monitoring node, multiple intermediate links are used and packets are exchanged in a Multi-hop manner. Discovered links in WSN and selected route for data exchange from a source to sink is shown in figure 6 and 7 respectively.

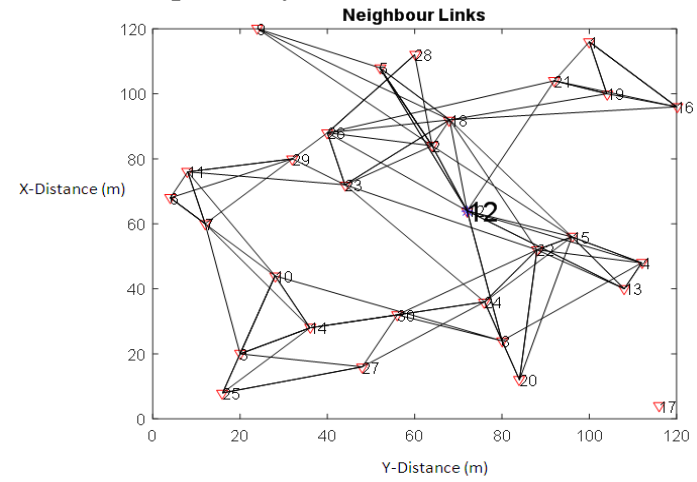

Figure 6: Discovered links from each node

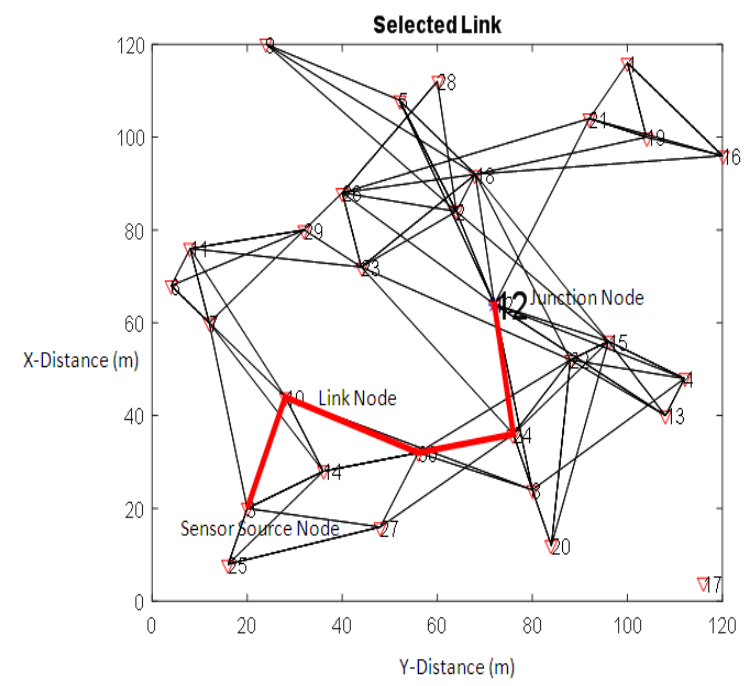

Figure 7: Selected route for data exchange in the network

Each of the communicated packets is exchanged over Multi-hop nodes to junction node, where these communicated packets are modulated to optical signals and transmitted over optical fiber network to the destination. In Optical Communication Modulation parameters considered is listed in table 2 .

Table 2: Parameter for optical modulation

\begin{tabular}{|l|l|}
\hline \multicolumn{1}{|c|}{ Parameters } & \multicolumn{1}{c|}{ Values } \\
\hline $\begin{array}{l}\text { Number of signal photons generated per } \\
\text { bit }\end{array}$ & 30 \\
\hline Number of Noise Photon generated per bit & 8 \\
\hline charge of electron, e & $1.6 \times \mathrm{e}^{-19} \mathrm{C}$ \\
\hline bit rate, $\mathrm{t}$ & $0.2 \times \mathrm{e}^{-16} \mathrm{sec}$ \\
\hline fraction of charge recombining, $\varepsilon$ & 0.8 \\
\hline band gap , $\mathrm{w}_{\mathrm{g}}$ & 0.95 \\
\hline Wavelength, $\lambda$ & $\left(1.24 / \mathrm{w}_{\mathrm{g}}\right) \times 10^{-6} ;$ \\
\hline Quantum efficiency & $0.9 ;$ \\
\hline Planck constant, $\mathrm{h}$ & $6.63 \times \mathrm{e}^{-34} ;$ \\
\hline Boltzmann's constant & $1.38 \times 10^{-23} ;$ \\
\hline speed of light, c & $3 \times 10^{8} \mathrm{~m} / \mathrm{sec} ;$ \\
\hline Dark current, $\mathrm{I}_{\mathrm{d}}$ & $10 \times 10^{-9} ;$ \\
\hline Load resistance, $\mathrm{R}_{\mathrm{l}}$ & $50 \mathrm{ohms} ;$ \\
\hline Bandwidth , bw & $35 \times 10^{12} \mathrm{~Hz} ;$ \\
\hline
\end{tabular}




\begin{tabular}{|l|l|}
\hline Thermal Noise Temperature & $200 \mathrm{~K}$ \\
\hline Amplifier Gain ,G & 2 \\
\hline driving current, drv_i & $(\mathrm{ns} \times \mathrm{e}) / \mathrm{t} ;$ \\
\hline
\end{tabular}

The modulated signal from the transmitter point is observed as shown in figure 8.

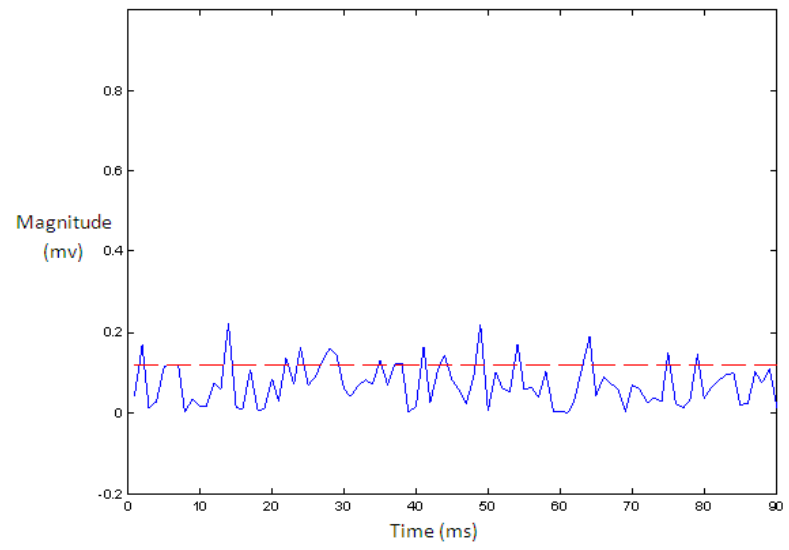

Figure 8: Optical Modulated Signal

The signal is distorted by the processing noise, and electrical discharges. The noise affected signal is shown in figure 9.

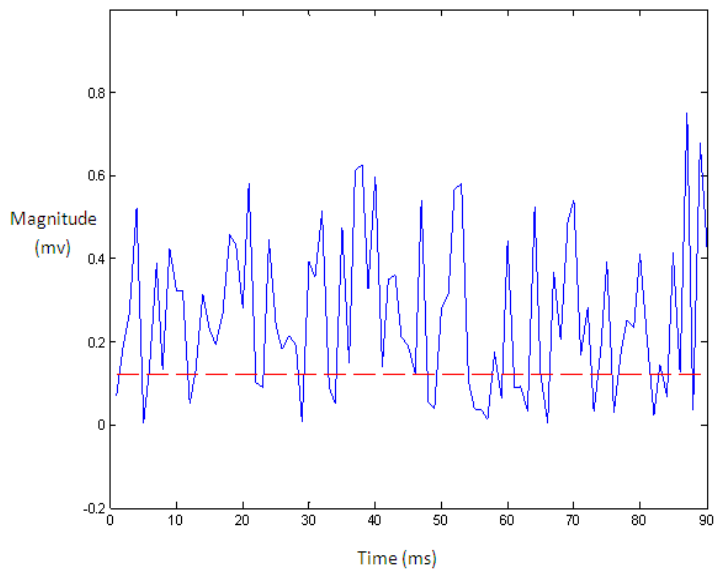

Figure 9: Noise effected signal

At receiver the captured signal is sampled to discrete coefficient for given number of samples per observation. Figure 10, 11 illustrates the detected coefficient for number of sample $=5$ for the threshold based and majority based detection logic respectively.

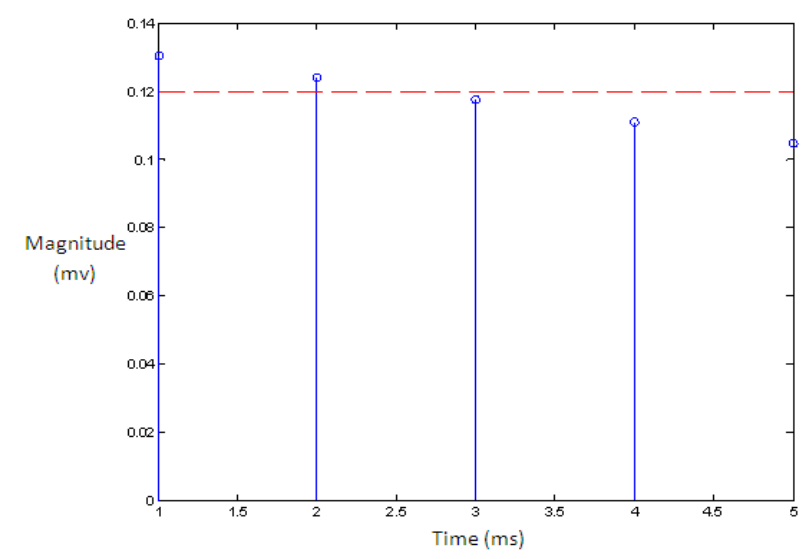

Figure 10: Threshold based detection

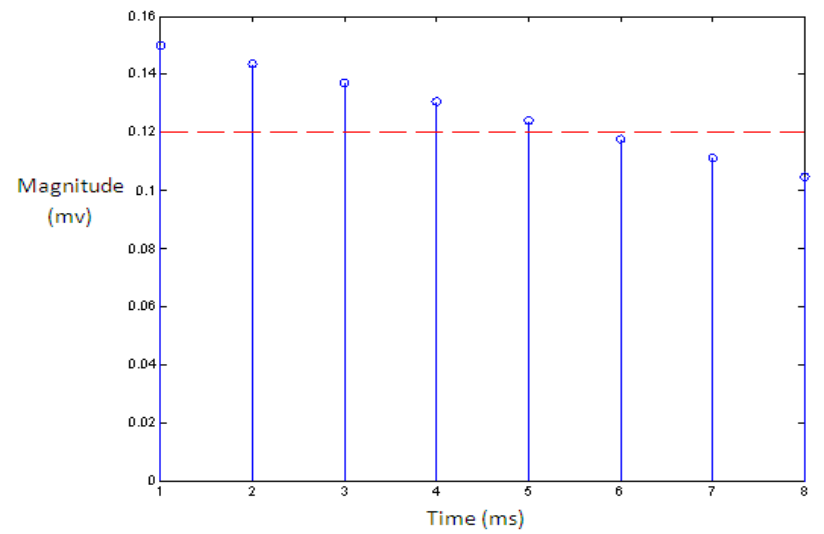

Figure 11: Majority based estimation

A similar detection for number of sample, $\mathrm{N}=8$ is presented in figure 12 .

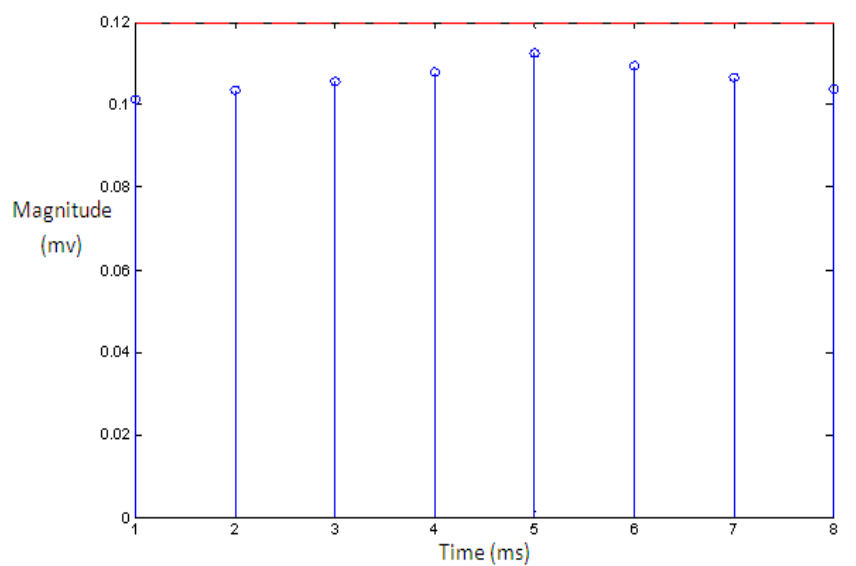

(a) 


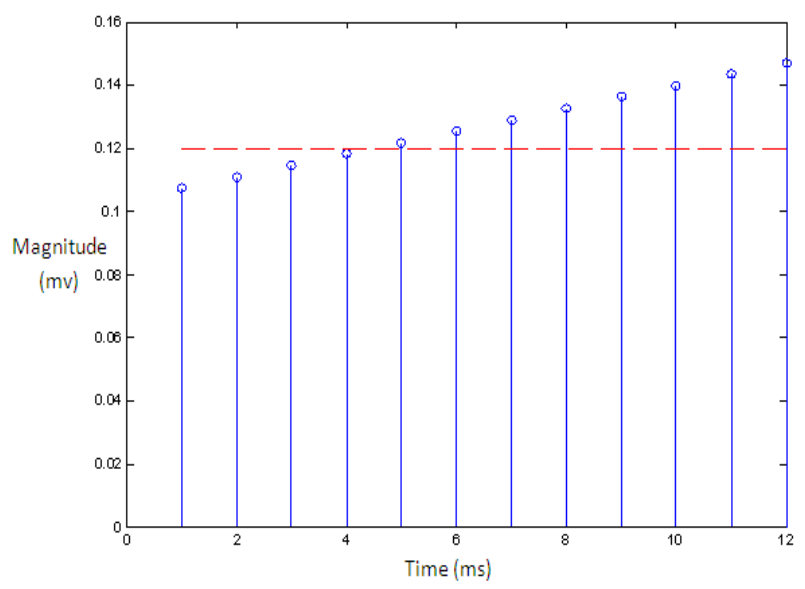

(b)

Figure 12: Decision for $\mathrm{N}=8$ using Majority decision logic for (a) logic ' 1 ' and (b) logic ' 0 '

The error performance of the developed system is as presented in figure 13.

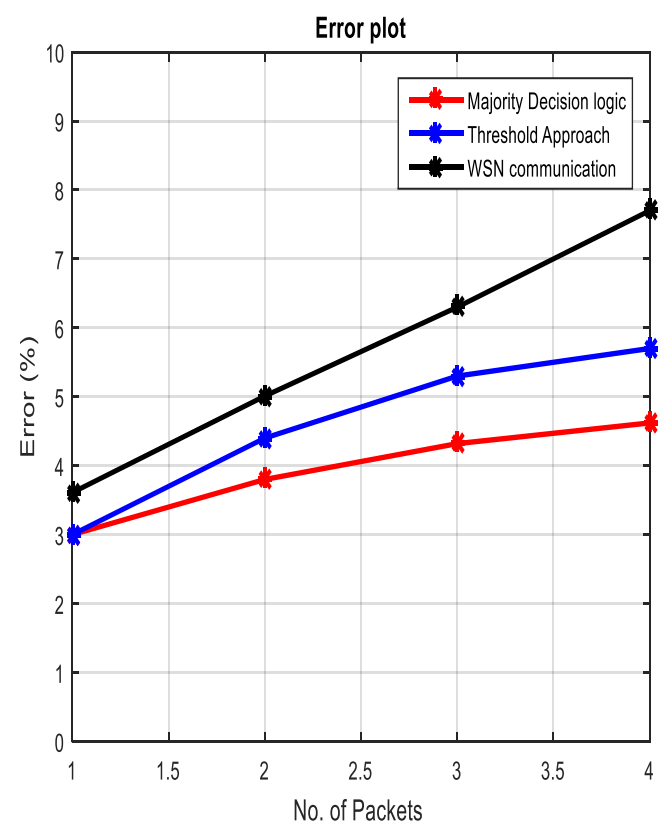

Figure 13: Error analysis of the simulated system

The error is minimized by the majority count operation, where the decision is made based on the density of the signal coefficient potentials.

The system throughput for the developed system is improved and there is successful communication of transmitted bits. The accuracy of detection increases the detection probability and hence the volume of data processed for an observing time interval increases. The system throughput for the developed approaches is shown in figure 14 .

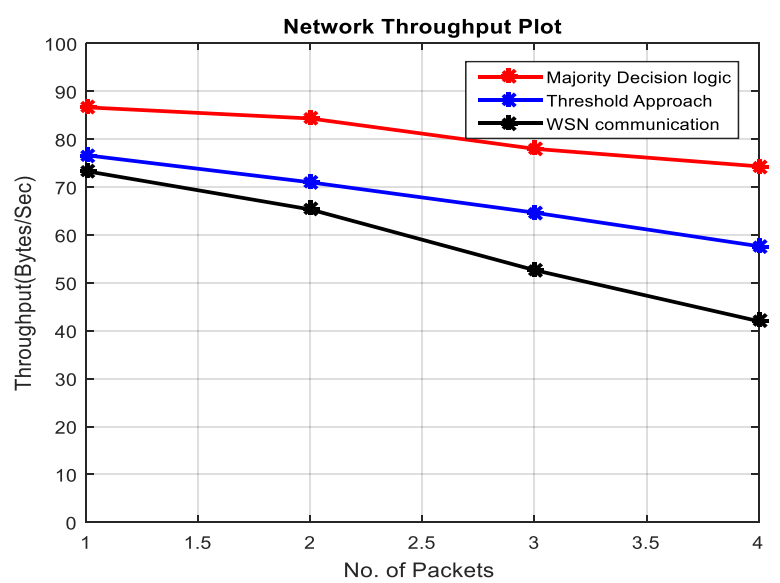

Figure 14: System throughput for the developed approaches

As the system throughput increases, the delay factor in the network reduces. The delay metric is defined as the total time taken for processing of given data over the communicating network. Figure 15 illustrates the delay performance metric of the developed approaches.

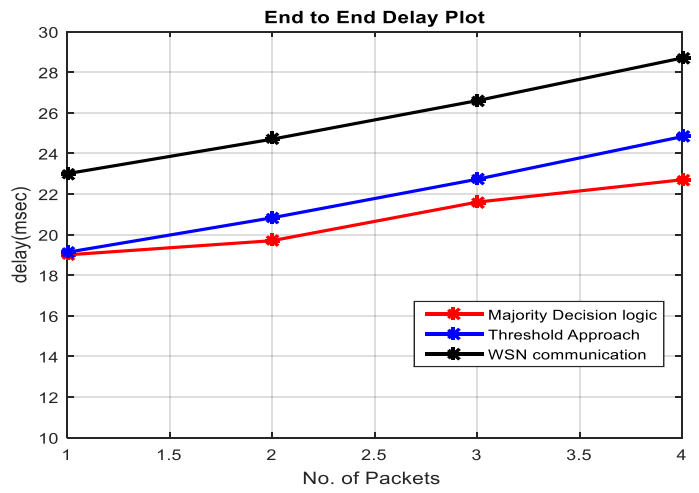

Figure 15: Delay performance of the system

The processing overhead defined as the number of processing coefficient buffered per node per cycle. Processing overhead at a link node is measured for the developed approach as shown in figure 16.

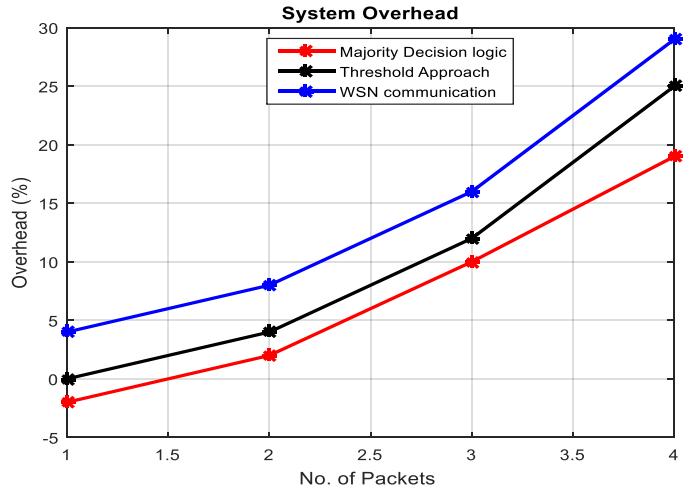

Figure 16: Processing overhead for the developed approaches

\section{CONCLUSION}

Wireless sensor network has a significant application in real time data monitoring and control operation. Wherein the remote monitoring has increased in recent past, the need of 
precision and high throughput has increased. Towards developing of method for throughput enhancement and accuracy improvement, in this paper an approach using majority based decision logic is proposed. A hybrid model of WSN integrated with optical communication is proposed. This approach defines an interference minimization and throughput enhancement by developing a decision logic using majority count value of discrete coefficient rather to hard decision threshold approach. The error performance and observed metrics illustrated the enhancement of proposed approach over hard threshold and standalone WSN communication.

\section{REFERENCES}

1. S Zahurul, N.Mariun, I.V.Grozescu, HanamotoTsuyoshi, Yasunori Mitani, M.L. Othman, H.Hizam, I.Z.Abidin, "Future strategic plan analysis for integrating distributed renewable generation to smart grid through wireless sensor network: Malaysia prospect", Renewable and Sustainable Energy Reviews Vol. 53, pp-978-992, 2016. https://doi.org/10.1016/j.rser.2015.09.020

2. L Alwis, T.Sun, K.T.V.Grattan, "Developments in optical fibre sensors for industrial applications", Optics \&LaserTechnology, Vol.78, pp-62-66, 2016. https://doi.org/10.1016/j.optlastec.2015.09.004

3. Joel Villatoron, JosebaZubia, "New perspectives in photonic crystal fibre sensors", Optics \&LaserTechnology, Vol.78, pp-67-75, 2016.

4. A. Xenakis , F. Foukalas , G. Stamoulis, " Cross-layer energy-aware topology control through Simulated Annealing for WSNs", Computers and Electrical Engineering pp-1-15, 2016. https://doi.org/10.1016/j.compeleceng.2016.02.015

5. Zilong Liao, Deshi Li, and Jian Chen, "A Handshake Based Ordered Scheduling MAC Protocol for Underwater Acoustic Local Area Networks", HINDAWI International Journal of Distributed Sensor Networks, 2015.

6. Guodong Sun, GuofuQiao and Lin Zhao, "Efficient link scheduling for rechargeable wireless ad hoc and sensor networks", EURASIP Journal on Wireless Communications and Networking 2013. https://doi.org/10.1186/1687-1499-2013-223

7. Harshita Chaurasiya, Dr. Shivnath Ghosh, "Performance Evaluation of Energy-Efficient Cluster based Algorithms in Wireless Sensor Network", International Journal of Advanced Trends in Computer Science and Engineering (IJATCSE), Vol.7, No.5, pp.78-81, 2018. https://doi.org/10.30534/ijatcse/2018/0375201

8. M. Yousof Naderi, Prusayon Nintanavongsa, and Kaushik R. Chowdhury, "RF-MAC: A Medium Access Control Protocol for Re-Chargeable Sensor Networks Powered by Wireless Energy Conservation", IEEE transactions on wireless communications, July 2014.
9. Jim A. Simpson, William C. Cox, John R. Krier, Brandon Cochenour, Brian L. Hughes and John F. Muth, "5 Mbps Optical Wireless Communication with Error Correction Coding for Underwater Sensor Nodes", IEEE, 2010.

10. Zahir Ahmad and Roger Green, "Link Design for Multi-hop Underwater Optical Wireless Sensor Network", Seventh International Conference on Systems and Networks Communications, ICSNC 2012.

11. Nasir Saeed, Abdulkadir Celik, Tareq Y. Al-Naffouri, and Mohamed-Slim Alouini, "Localization of Energy Harvesting Empowered Underwater Optical Wireless Sensor Networks", IEEE ICASSP 2018.

12. Na Zhang, "An Algorithm of Wireless Sensor Network Based on Free Space Optical Communication", International Journal of Online and Biomedical Engineering (IJOE), Vol. 13, No. 7, 2017.

13. Nathan J. Gomes, Maria Morant, Arokiaswami Alphones, Béatrice Cabon, John E. Mitchell, Christophe Lethien, Mark Csörnyei, Andreas Stöhr, and Stavros Iezekiel, "Radio-over-fiber transport for the support of wireless broadband services", Journal of Optical Networking, Vol. 8, No. 2, February 2009.

https://doi.org/10.1364/JON.8.000156

14. M. I. Afzal, Waqar Mahmood, Sheikh Muhammad Sajid and Shin Seoyong, "Optical Wireless Communication and Recharging Mechanism of Wireless Sensor Network by Using CCRs" International Journal of Advanced Science and Technology, Volume 13, December, 2009.

15. Hany Elgala, Raed Mesleh, Harald Haas, "Indoor Optical Wireless Communication: Potential and State-of-the-Art", IEEE Communications Magazine, September 2011. https://doi.org/10.1109/MCOM.2011.6011734

16. Heejin Lee, Yeonjoo Kim, Kyungrak Sohn, "Optical Wireless Sensor Networks Based on VLC with PLC-Ethernet Interface", International Journal of Electronics and Communication Engineering Vol:5, No:9, 2011.

17. Jatuporn Chinrungrueng Udomporn Sunantachaikul Satien Triamlumlerd, "Smart Parking: an Application of opticalWireless Sensor Network", International Symposium on applications and the Internet Workshops, 2017.

18. Shota Teramoto, Tomoaki Ohtsuki, "Optical Wireless Sensor Network System Using Corner Cube Retro reflectors", EURASIP Journal on Applied Signal Processing 2005:1, 39-44

19. Shuo Yang, Bochao Zhou, Tong Sun, Kenneth T.V. Grattan, "A Novel Optical Sensor Platform Designed for Wireless Sensor Networks", Journal of Physics: Conference Series 450 (2013) 012007. https://doi.org/10.1088/1742-6596/450/1/012007

20. Yuan Li, Nikolaos Pappas, Vangelis Angelakis, Michal Pioro and Di Yuan, "Optimization of Free Space Optical Wireless Network for Cellular 
Backhauling", 2015, IEEE Journal on Selected Areas in Communications, (33), 9, 1841-1854. 2015.

https://doi.org/10.1109/JSAC.2015.2432518

21. A Nageswar Rao, B Rajendra Naik, L Nirmala Devi, "Location Aware Relay Node Placement for Energy Constrained Wireless Sensor Networks", International Journal of Advanced Trends in Computer Science and Engineering (IJATCSE), Vol.8, No.3, pp.519-526,2019.

https://doi.org/10.30534.ijatcse/2019/28832019.

22. S Taruna, MeghaR.Tiwari and Sakshi Shringi, "Event Driven Routing Protocols for Wireless Sensor Network- A Survey", International Journal on Computational Sciences \& Applications (IJCSA) 2013. 